\title{
$31 \quad$ ILLICIT AND BENZODIAZEPINE DRUGS USE AMONG FATALLY INJURED DRIVERS IN URBAN AREAS OF KUALA LUMPAR
}

doi:10.1136/injuryprev-2012-040580c.31

${ }^{1} \mathrm{~N}$ Mohamed*, ${ }^{1} \mathrm{~W}$ Ameer-Batcha, ${ }^{2} \mathrm{NK}$ Abdullah, ${ }^{3} \mathrm{~F}$ Yusoff. ${ }^{1}$ Malaysian Institute of Road Safety Research, Malaysia; ${ }^{2}$ National Institute of Forensic Medicine, Malaysia; ${ }^{3}$ Institute of Public Health, Malaysia

Background Driving under the influence of drugs is one of the welldocumented risk factors for road traffic fatality. To date, in Malaysia there is no published report on the incidence of fatally injured driver who involved in road crashes positive for illicit and medicinal drug.

Aim/Objectives The aim of the study was to determine the incidence of illicit and benzodiazepine drugs use among fatally injured drivers who involved in road traffic crashes.

Methods A retrospective cross-sectional study was conducted based on post mortem and toxicological files retrieved from the Department of Forensic Medicine, at a local hospital in urban area of Kuala Lumpur.

Results Three hundred and ninety-one (391) fatally injured drivers were eligible for analysis of drugs use. The study revealed that the incidence of fatal drivers positive for illicit drugs use was $10 \%$ with opiates group at the top of the list (5.4\%) followed by amphetamines $(2.8 \%)$, cannabis $(1.02 \%)$ and ketamine $(0.8 \%)$. For medicinal drug, benzodiazepines group was at the top of the list $(6.9 \%)$ followed by anti-histamine (0.8\%) and anti-epileptic drug (0.3\%).

Significance/Contribution to the Field These findings highlight the need to focus on prevention activities related to driving under the influenced of illicit drugs as part of the overall strategies for road safety prevention. For Benzodiazepine drug, this finding carry very important message for prevention of crashes especially to the medical practitioners that used to prescribes benzodiazepines to their patients. Special emphasise on the choice of short, medium and long acting benzodiazepines drugs and warning regarding its impact on a person's ability to drive safely need to be given to their patients. This is truly important, as driving under the influenced of benzodiazepines drugs cannot be enforced at the roadside. 